\title{
Fully Relativistic Calculations of Magneto-Optical Kerr Effect
}

\author{
Ming-Fang Li, Toshihiro AriIzumi, and Shugo Suzuki \\ Institute of Materials Science, University of Tsukuba, Tsukuba 305-8573
}

(Received February 23, 2007)

\begin{abstract}
We study the magneto-optical Kerr effect using fully relativistic calculations. Spin-orbit coupling is dealt with exactly solving the Dirac equation directly and the matrix elements of the Dirac matrices $\boldsymbol{\alpha}$ are used in a fully relativistic expression of the Kubo formula for the optical conductivity derived with a relativistic sum rule. We also perform approximate calculations of the optical conductivity to examine the accuracy of a partly relativistic expression in which the matrix elements of the momentum operator $\boldsymbol{p}$ are used instead. As an example, we carry out calculations for bcc Fe and fcc Ni using the fully relativistic full-potential linearcombination-of-atomic-orbitals method. It is found that the partly relativistic treatment is good for the diagonal optical conductivity while it is not very good for the off-diagonal optical conductivity, the Kerr rotation angle, and the Kerr ellipticity. The results of the present study are compared to those of experimental and other theoretical studies.
\end{abstract}

KEYWORDS: magneto-optical Kerr effect, fully relativistic calculation, full-potential calculation, LCAO method, spin-orbit coupling, relativistic sum rule, optical conductivity, $\mathrm{Fe}, \mathrm{Ni}$

\section{Introduction}

In the last few decades, the magneto-optical Kerr effect (MOKE) has attracted much attention because of its application in data storage technology. ${ }^{1,2)}$ The microscopic origin of the MOKE is a combination of spin-orbit coupling and the magnetization of a material. The effect is usually not very large; for example, the Kerr rotation angles are less than 1 degree for the ferromagnetic $3 d$ transition metals, Fe, Co, and Ni. For this reason, careful measurements in experimental studies and accurate calculations in theoretical studies are indispensable. ${ }^{3-7)}$

To study the MOKE theoretically, first-principles calculations based on the density functional theory have been carried out; the full-potential linear muffin-tin orbital method, the augmented spherical wave method, and the full-potential linear augmented plane wave method are used as powerful tools for this purpose. ${ }^{8-26)}$ Since the MOKE is sensitive to the details of the electronic structure of a material, careful analysis is indispensable; for example, fullpotential approach, inclusion of relativistic effects, accurate evaluation of matrix elements, etc. The recent theoretical progress enables a precise investigation of the MOKE and a detailed comparison between calculated and measured Kerr spectra becomes possible.

There still remain, however, a few points to be examined theoretically. One is that fully 
relativistic calculations in the sense that the Dirac equation is solved directly have not been performed yet; so far, spin-orbit coupling has been dealt with using a second variation procedure. Another is that a fully relativistic expression of the Kubo formula for the optical conductivity in the sense that the matrix elements of the Dirac matrices $\boldsymbol{\alpha}$ are used has not been employed yet; so far, the matrix elements have been approximated with those of the momentum operator $\boldsymbol{p}=-i \hbar \nabla \cdot{ }^{27,28)}$ These points can be examined employing the fully relativistic full-potential linear-combination-of-atomic-orbitals (FFLCAO) method. ${ }^{29)}$

The purpose of the present study is to investigate the MOKE using the FFLCAO method to deal with spin-orbit coupling exactly and also to evaluate the matrix elements of the Dirac matrices $\boldsymbol{\alpha}$ necessary for calculating the optical conductivity with a fully relativistic expression of the Kubo formula derived with a relativistic sum rule. As an example, we calculate the optical conductivity and the Kerr spectra of bcc Fe and fcc Ni. In $\S 2$, we derive the relativistic sum rule within the independent electron approximation and, in $\S 3$, we apply it to the derivation of the fully relativistic expression of the Kubo formula for the optical conductivity. The results and discussion of the calculations for bcc Fe and fcc Ni are given in $\S 4$. Finally, we give the conclusions of the present study in $\S 5$.

\section{Relativistic sum rule}

We now derive a relativistic sum rule within the independent electron approximation, which will be applied to the derivation of the fully relativistic expression of the Kubo formula for the optical conductivity in the next section.

The Dirac equation is given as

$$
\left[c \boldsymbol{\alpha} \cdot \boldsymbol{p}+(\beta-1) m c^{2}+V\right]|\nu\rangle=\varepsilon_{\nu}|\nu\rangle,
$$

where $\boldsymbol{\alpha}$ and $\beta$ are the Dirac matrices and $V$ is the $4 \times 4$ one-electron potential. For a crystalline solid, the quantum number $\nu$ represents the band index $n$ and the wave number vector $\boldsymbol{k}$. In the following, the Dirac Hamiltonian is denoted by $H$, i.e.,

$$
H=c \boldsymbol{\alpha} \cdot \boldsymbol{p}+(\beta-1) m c^{2}+V .
$$

The solutions of eq.(1) are classified into two groups; one is a group such that, in the nonrelativistic limit, i.e., in the limit of $c \rightarrow \infty$, the solutions in this group converge to the corresponding solutions of the two-component Schrödinger equation while the other is a group such that the energies of the solutions in this group become $-\infty$ in the nonrelativistic limit. In the present study, we refer to the former solutions as the positive energy states and also refer to the latter solutions as the negative energy states regardless of whether $\varepsilon_{\nu}$ is positive or negative.

The Hamiltonian and the ground state of the many-electron system in a solid are then 
given by

$$
\mathcal{H}=\sum_{\nu} \varepsilon_{\nu} a_{\nu}^{\dagger} a_{\nu}
$$

and

$$
\left.|\mathrm{G}\rangle=\prod_{\nu}^{\mathrm{occ}} a_{\nu}^{\dagger} \mid \text { null }\right\rangle,
$$

respectively. In the above equations, $a_{\nu}^{\dagger}$ and $a_{\nu}$ are the creation and annihilation operators of the electron in the one-electron state $|\nu\rangle$, respectively. Also, in eq.(4), the product is over the occupied states including all the negative energy states; in this equation, |null $\rangle$ represents the state in which there are no electrons in either the positive or negative energy states.

We now consider the following operators:

$$
\mathcal{X}=\sum_{\nu \mu}\langle\nu|\xi| \mu\rangle a_{\nu}^{\dagger} a_{\mu}
$$

and

$$
\mathcal{Y}=\sum_{\nu \mu}\langle\nu|\eta| \mu\rangle a_{\nu}^{\dagger} a_{\mu}
$$

where $\xi$ and $\eta$ represent the $x, y$, or $z$ coordinate of an electron. It is straightforward to show

$$
[\mathcal{X}, \mathcal{H}]=\sum_{\nu \mu} i \hbar c\left\langle\nu\left|\alpha_{\xi}\right| \mu\right\rangle a_{\nu}^{\dagger} a_{\mu}
$$

if one notices

$$
[\xi, H]=i \hbar c \alpha_{\xi}
$$

Furthermore, we can derive the following commutation relation:

$$
[\mathcal{Y},[\mathcal{X}, \mathcal{H}]]=0
$$

The derivation is also straightforward because

$$
\left[\eta, \alpha_{\xi}\right]=0
$$

Next, we consider the expectation value of eq.(9) with respect to the ground state $|G\rangle$ as follows:

$$
\langle\mathrm{G}|[\mathcal{Y},[\mathcal{X}, \mathcal{H}]]| \mathrm{G}\rangle=0 .
$$

On the other hand, the left-hand side of the above equation is evaluated as

$$
\langle\mathrm{G}|[\mathcal{Y},[\mathcal{X}, \mathcal{H}]]| \mathrm{G}\rangle=\sum_{\nu \mu} f_{\nu}\left(1-f_{\mu}\right)\left(\varepsilon_{\nu}-\varepsilon_{\mu}\right)(\langle\nu|\xi| \mu\rangle\langle\mu|\eta| \nu\rangle+\langle\nu|\eta| \mu\rangle\langle\mu|\xi| \nu\rangle),
$$

where $f_{\nu}$ and $f_{\mu}$ represent the Fermi distribution function. Calculating the matrix element of eq.(8), we obtain

$$
\left(\varepsilon_{\mu}-\varepsilon_{\nu}\right)\langle\nu|\xi| \mu\rangle=i \hbar c\left\langle\nu\left|\alpha_{\xi}\right| \mu\right\rangle
$$


Accordingly, eq.(12) becomes

$$
\langle\mathrm{G}|[\mathcal{Y},[\mathcal{X}, \mathcal{H}]]| \mathrm{G}\rangle=\hbar^{2} c^{2} \sum_{\nu \mu} f_{\nu}\left(1-f_{\mu}\right)\left[\frac{\left\langle\nu\left|\alpha_{\xi}\right| \mu\right\rangle\left\langle\mu\left|\alpha_{\eta}\right| \nu\right\rangle}{\varepsilon_{\nu}-\varepsilon_{\mu}}+\frac{\left\langle\nu\left|\alpha_{\eta}\right| \mu\right\rangle\left\langle\mu\left|\alpha_{\xi}\right| \nu\right\rangle}{\varepsilon_{\nu}-\varepsilon_{\mu}}\right] .
$$

We thus arrive at the following relativistic sum rule:

$$
\sum_{\nu \mu} f_{\nu}\left(1-f_{\mu}\right)\left[\frac{\left\langle\nu\left|\alpha_{\xi}\right| \mu\right\rangle\left\langle\mu\left|\alpha_{\eta}\right| \nu\right\rangle}{\varepsilon_{\nu}-\varepsilon_{\mu}}+\frac{\left\langle\nu\left|\alpha_{\eta}\right| \mu\right\rangle\left\langle\mu\left|\alpha_{\xi}\right| \nu\right\rangle}{\varepsilon_{\nu}-\varepsilon_{\mu}}\right]=0 .
$$

\section{Optical conductivity}

The fully relativistic expression of the Kubo formula for the optical conductivity due to the interband transition can be obtained as ${ }^{30)}$

$$
\sigma_{\xi \eta}(\omega)=\frac{i e^{2} c^{2}}{\Omega \omega_{\tau}} \sum_{\nu \mu} f_{\nu}\left(1-f_{\mu}\right)\left[\frac{\left\langle\nu\left|\alpha_{\xi}\right| \mu\right\rangle\left\langle\mu\left|\alpha_{\eta}\right| \nu\right\rangle}{\varepsilon_{\nu}-\varepsilon_{\mu}+\hbar \omega_{\tau}}+\frac{\left\langle\nu\left|\alpha_{\eta}\right| \mu\right\rangle\left\langle\mu\left|\alpha_{\xi}\right| \nu\right\rangle}{\varepsilon_{\nu}-\varepsilon_{\mu}-\hbar \omega_{\tau}}\right] .
$$

Here, $\Omega$ is the volume of a solid and $\omega_{\tau}$ represents $\omega+i / \tau, \omega$ being the frequency of light and $\tau$ being phenomenological relaxation time. In eq.(16), the summation over $\nu$ is for both the positive and negative energy states while the summation over $\mu$ is only for the positive energy states because of the factor $1-f_{\mu}$. Although eq.(16) is formally correct, the summation involving the negative energy states causes a serious difficulty when calculating the optical conductivity with this expression. In this section, we eliminate the summation involving the negative energy states using the relativistic sum rule derived in the previous section.

We first decompose the summation over $\nu$ into two parts; one is for the positive energy states and the other is for the negative energy states as follows:

$$
\begin{aligned}
\sigma_{\xi \eta}(\omega)= & \frac{i e^{2} c^{2}}{\Omega \omega_{\tau}} \sum_{\nu}{ }^{(+)} \sum_{\mu}{ }^{(+)} f_{\nu}\left(1-f_{\mu}\right)\left[\frac{\left\langle\nu\left|\alpha_{\xi}\right| \mu\right\rangle\left\langle\mu\left|\alpha_{\eta}\right| \nu\right\rangle}{\varepsilon_{\nu}-\varepsilon_{\mu}+\hbar \omega_{\tau}}+\frac{\left\langle\nu\left|\alpha_{\eta}\right| \mu\right\rangle\left\langle\mu\left|\alpha_{\xi}\right| \nu\right\rangle}{\varepsilon_{\nu}-\varepsilon_{\mu}-\hbar \omega_{\tau}}\right] \\
& +\frac{i e^{2} c^{2}}{\Omega \omega_{\tau}} \sum_{\nu}{ }^{(-)} \sum_{\mu}{ }^{(+)} f_{\nu}\left(1-f_{\mu}\right)\left[\frac{\left\langle\nu\left|\alpha_{\xi}\right| \mu\right\rangle\left\langle\mu\left|\alpha_{\eta}\right| \nu\right\rangle}{\varepsilon_{\nu}-\varepsilon_{\mu}+\hbar \omega_{\tau}}+\frac{\left\langle\nu\left|\alpha_{\eta}\right| \mu\right\rangle\left\langle\mu\left|\alpha_{\xi}\right| \nu\right\rangle}{\varepsilon_{\nu}-\varepsilon_{\mu}-\hbar \omega_{\tau}}\right],
\end{aligned}
$$

where $\sum^{(+)}$denotes the summation over the positive energy states and $\sum^{(-)}$denotes the summation over the negative energy states. Furthermore, since the energy differences between the negative energy states and the positive energy states are very large in comparison to the photon energy, it is a very good approximation to ignore $\hbar \omega_{\tau}$ in the summation involving both the positive and negative energy states:

$$
\begin{aligned}
\sigma_{\xi \eta}(\omega)= & \frac{i e^{2} c^{2}}{\Omega \omega_{\tau}} \sum_{\nu}{ }^{(+)} \sum_{\mu}{ }^{(+)} f_{\nu}\left(1-f_{\mu}\right)\left[\frac{\left\langle\nu\left|\alpha_{\xi}\right| \mu\right\rangle\left\langle\mu\left|\alpha_{\eta}\right| \nu\right\rangle}{\varepsilon_{\nu}-\varepsilon_{\mu}+\hbar \omega_{\tau}}+\frac{\left\langle\nu\left|\alpha_{\eta}\right| \mu\right\rangle\left\langle\mu\left|\alpha_{\xi}\right| \nu\right\rangle}{\varepsilon_{\nu}-\varepsilon_{\mu}-\hbar \omega_{\tau}}\right] \\
& +\frac{i e^{2} c^{2}}{\Omega \omega_{\tau}} \sum_{\nu}{ }^{(-)} \sum_{\mu}{ }^{(+)} f_{\nu}\left(1-f_{\mu}\right)\left[\frac{\left\langle\nu\left|\alpha_{\xi}\right| \mu\right\rangle\left\langle\mu\left|\alpha_{\eta}\right| \nu\right\rangle}{\varepsilon_{\nu}-\varepsilon_{\mu}}+\frac{\left\langle\nu\left|\alpha_{\eta}\right| \mu\right\rangle\left\langle\mu\left|\alpha_{\xi}\right| \nu\right\rangle}{\varepsilon_{\nu}-\varepsilon_{\mu}}\right] .
\end{aligned}
$$


Rewriting the relativistic sum rule derived in the previous section as

$$
\begin{aligned}
& \sum_{\nu}{ }^{(+)} \sum_{\mu}{ }^{(+)} f_{\nu}\left(1-f_{\mu}\right)\left[\frac{\left\langle\nu\left|\alpha_{\xi}\right| \mu\right\rangle\left\langle\mu\left|\alpha_{\eta}\right| \nu\right\rangle}{\varepsilon_{\nu}-\varepsilon_{\mu}}+\frac{\left\langle\nu\left|\alpha_{\eta}\right| \mu\right\rangle\left\langle\mu\left|\alpha_{\xi}\right| \nu\right\rangle}{\varepsilon_{\nu}-\varepsilon_{\mu}}\right] \\
& +\sum_{\nu}{ }^{(-)} \sum_{\mu}{ }^{(+)} f_{\nu}\left(1-f_{\mu}\right)\left[\frac{\left\langle\nu\left|\alpha_{\xi}\right| \mu\right\rangle\left\langle\mu\left|\alpha_{\eta}\right| \nu\right\rangle}{\varepsilon_{\nu}-\varepsilon_{\mu}}+\frac{\left\langle\nu\left|\alpha_{\eta}\right| \mu\right\rangle\left\langle\mu\left|\alpha_{\xi}\right| \nu\right\rangle}{\varepsilon_{\nu}-\varepsilon_{\mu}}\right]=0,
\end{aligned}
$$

we obtain

$$
\begin{aligned}
\sigma_{\xi \eta}(\omega)= & \frac{i e^{2} c^{2}}{\Omega \omega_{\tau}} \sum_{\nu}{ }^{(+)} \sum_{\mu}{ }^{(+)} f_{\nu}\left(1-f_{\mu}\right) \\
& \times\left[\frac{\left\langle\nu\left|\alpha_{\xi}\right| \mu\right\rangle\left\langle\mu\left|\alpha_{\eta}\right| \nu\right\rangle}{\varepsilon_{\nu}-\varepsilon_{\mu}+\hbar \omega_{\tau}}+\frac{\left\langle\nu\left|\alpha_{\eta}\right| \mu\right\rangle\left\langle\mu\left|\alpha_{\xi}\right| \nu\right\rangle}{\varepsilon_{\nu}-\varepsilon_{\mu}-\hbar \omega_{\tau}}-\frac{\left\langle\nu\left|\alpha_{\xi}\right| \mu\right\rangle\left\langle\mu\left|\alpha_{\eta}\right| \nu\right\rangle}{\varepsilon_{\nu}-\varepsilon_{\mu}}-\frac{\left\langle\nu\left|\alpha_{\eta}\right| \mu\right\rangle\left\langle\mu\left|\alpha_{\xi}\right| \nu\right\rangle}{\varepsilon_{\nu}-\varepsilon_{\mu}}\right] .
\end{aligned}
$$

It should be noted that the above equation involves only the positive energy states and thus one can employ usual computational techniques for the calculations with this expression. Finally, after manipulating eq.(20), we obtain the fully relativistic expression of the Kubo formula for the optical conductivity:

$$
\begin{aligned}
\sigma_{\xi \eta}(\omega)= & \frac{2 i e^{2} c^{2}}{\hbar \Omega} \sum_{\nu}{ }^{(+)} \sum_{\mu}{ }^{(+)} \frac{f_{\nu}\left(1-f_{\mu}\right)}{\omega_{\tau}^{2}-\omega_{\mu \nu}^{2}} \\
& \times\left\{\frac{\omega_{\tau}}{\omega_{\mu \nu}} \operatorname{Re}\left[\left\langle\nu\left|\alpha_{\xi}\right| \mu\right\rangle\left\langle\mu\left|\alpha_{\eta}\right| \nu\right\rangle\right]+i \operatorname{Im}\left[\left\langle\nu\left|\alpha_{\xi}\right| \mu\right\rangle\left\langle\mu\left|\alpha_{\eta}\right| \nu\right\rangle\right]\right\},
\end{aligned}
$$

where $\omega_{\mu \nu}$ denotes $\left(\varepsilon_{\mu}-\varepsilon_{\nu}\right) / \hbar$, or, for a crystalline solid,

$$
\begin{aligned}
\sigma_{\xi \eta}(\omega)= & \frac{2 i e^{2} c^{2}}{\hbar \Omega} \sum_{\boldsymbol{k}} \sum_{l}{ }^{(+)} \sum_{n}{ }^{(+)} \frac{f_{l \boldsymbol{k}}\left(1-f_{n \boldsymbol{k}}\right)}{\omega_{\tau}^{2}-\omega_{n l \boldsymbol{k}}^{2}} \\
& \times\left\{\frac{\omega_{\tau}}{\omega_{n l \boldsymbol{k}}} \operatorname{Re}\left[\left\langle l \boldsymbol{k}\left|\alpha_{\xi}\right| n \boldsymbol{k}\right\rangle\left\langle n \boldsymbol{k}\left|\alpha_{\eta}\right| l \boldsymbol{k}\right\rangle\right]+i \operatorname{Im}\left[\left\langle l \boldsymbol{k}\left|\alpha_{\xi}\right| n \boldsymbol{k}\right\rangle\left\langle n \boldsymbol{k}\left|\alpha_{\eta}\right| l \boldsymbol{k}\right\rangle\right]\right\},
\end{aligned}
$$

where $\omega_{n l \boldsymbol{k}}$ denotes $\left(\varepsilon_{n \boldsymbol{k}}-\varepsilon_{l \boldsymbol{k}}\right) / \hbar$. Equation (22) is the final formula for the optical conductivity used in the present study. We also use a partly relativistic expression for comparison; in eq.(22), employing the Pauli approximation, ${ }^{31)}$ i.e., replacing the matrix element $\left\langle l \boldsymbol{k}\left|\alpha_{\xi}\right| n \boldsymbol{k}\right\rangle$ with $\left(l \boldsymbol{k}\left|p_{\xi} / m c\right| n \boldsymbol{k}\right)$, where $\left.\mid\right)$ is the large component of |\rangle in the standard representation and $p_{\xi}$ is the $\xi$ component of the momentum operator $\boldsymbol{p}$, we obtain the partly relativistic expression: ${ }^{27,28)}$

$$
\begin{aligned}
\sigma_{\xi \eta}(\omega)= & \frac{2 i e^{2}}{m^{2} \hbar \Omega} \sum_{\boldsymbol{k}} \sum_{l}{ }^{(+)} \sum_{n}{ }^{(+)} \frac{f_{l \boldsymbol{k}}\left(1-f_{n \boldsymbol{k}}\right)}{\omega_{\tau}^{2}-\omega_{n l \boldsymbol{k}}^{2}} \\
& \times\left\{\frac{\omega_{\tau}}{\omega_{n l \boldsymbol{k}}} \operatorname{Re}\left[\left(l \boldsymbol{k}\left|p_{\xi}\right| n \boldsymbol{k}\right)\left(n \boldsymbol{k}\left|p_{\eta}\right| l \boldsymbol{k}\right)\right]+i \operatorname{Im}\left[\left(l \boldsymbol{k}\left|p_{\xi}\right| n \boldsymbol{k}\right)\left(n \boldsymbol{k}\left|p_{\eta}\right| l \boldsymbol{k}\right)\right]\right\} .
\end{aligned}
$$

Using eq.(22) or eq.(23), the Kerr rotation angle $\theta_{\mathrm{K}}(\omega)$ and the Kerr ellipticity $\eta_{\mathrm{K}}(\omega)$ are 
given by

$$
\theta_{\mathrm{K}}(\omega)+i \eta_{\mathrm{K}}(\omega)=-\frac{\sigma_{x y}(\omega)}{\sigma_{x x}(\omega) \sqrt{1+i(4 \pi / \omega) \sigma_{x x}(\omega)}}
$$

\section{Computational details}

In the present study, we employed the FFLCAO method based on the density functional theory within the local-spin-density approximation. ${ }^{29)}$ Solving the Dirac-Kohn-Sham equations directly, spin-orbit coupling is taken into account exactly. Furthermore, we carried out calculations adopting the exchange-correlation energy functional within the local-spin-density approximation expressed by the Perdew-Zunger parameterization of Ceperley and Alder's results. ${ }^{32,33)}$

In the present study, we used the experimental lattice constants: $2.8663 \AA$ for bcc Fe and $3.5238 \AA$ for fcc Ni. We chose basis functions so that they have enough variational flexibility. That is, we used not only atomic orbitals of neutral atoms but also those of charged atoms. We used a single orbital for each core orbital and double orbitals for each valence orbital. Double valence orbitals are necessary for describing the contraction of atomic orbitals accompanied with cohesion. The atomic orbitals used for Fe are $1 s, 2 s, 2 p, 3 s, 3 p, 3 d$, and $4 s$ atomic orbitals of neutral $\mathrm{Fe}$ atoms and $3 d$ and $4 s$ atomic orbitals of $\mathrm{Fe}^{2+}$ atoms and $4 p$ atomic orbitals of $\mathrm{Fe}^{+}$and $\mathrm{Fe}^{3+}$ atoms. Also, the atomic orbitals used for Ni are $1 s, 2 s, 2 p, 3 s, 3 p, 3 d$, and $4 s$ atomic orbitals of neutral $\mathrm{Ni}$ atoms and $3 d$ and $4 s$ atomic orbitals of $\mathrm{Ni}^{2+}$ atoms and $4 p$ atomic orbitals of $\mathrm{Ni}^{+}$and $\mathrm{Ni}^{3+}$ atoms.

Real space integration is performed using the atomic partitioning method; ${ }^{34-36)}$ the number of mesh points per atom is 3096. Also, reciprocal space integration in the Brillouin zone is performed using the good-lattice-point method. ${ }^{37)}$ The number of $\boldsymbol{k}$ points used for selfconsistent calculations is 185 and that used for the calculations of the optical conductivity is up to $4044 \boldsymbol{k}$ points for checking the convergence. Furthermore, we performed the multipolar expansion of the electrostatic potential up to 3 . In calculating the optical conductivity, we use a phenomenological relaxation time $\hbar / \tau=0.5 \mathrm{eV}$ throughout and, to avoid ambiguity, we consider only the interband contribution to the optical conductivity given by eq.(22) or eq.(23), ignoring the intraband contribution described by an empirical Drude term.

\section{Results and discussion}

We first examine the computational error accompanied with the sampling of $\boldsymbol{k}$ points. To this end, we take the Kerr rotation angle of Fe as an example because this quantity is very sensitive to computational details. In Fig. 1, we show the spectra of the Kerr rotation angle calculated with $828 \boldsymbol{k}$ points, $1958 \boldsymbol{k}$ points, and $4044 \boldsymbol{k}$ points. It is found that the three results are in good agreement with each other at least between $1 \mathrm{eV}$ and $8 \mathrm{eV}$. We thus consider the results of calculations with $4044 \boldsymbol{k}$ points to be reliable in this energy range. In the following, we show the results of calculations with $4044 \boldsymbol{k}$ points. 


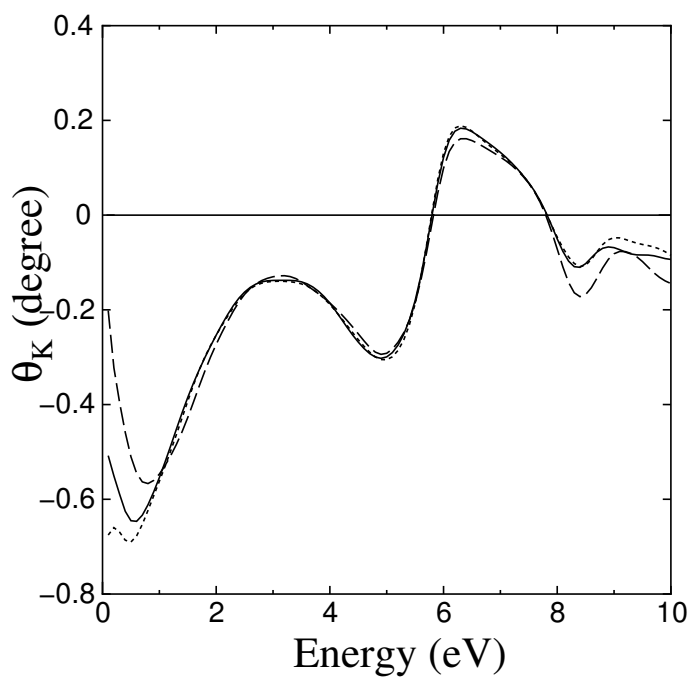

Fig. 1. Spectra of Kerr rotation angle for bcc Fe. The dashed line is the result calculated with 828 $\boldsymbol{k}$ points. The dotted line is the result calculated with $1958 \boldsymbol{k}$ points. The solid line is the result calculated with $4044 \boldsymbol{k}$ points.

We now show the diagonal optical conductivity, $\sigma_{x x}(\omega)$, of Fe in Fig. 2 and that of Ni in Fig. 3. Figures 2(a) and 3(a) represent $\operatorname{Re}\left[\sigma_{x x}(\omega)\right]$ and Figs. 2(b) and 3(b) represent $\operatorname{Im}\left[\sigma_{x x}(\omega)\right]$. In these figures, the solid lines show the results calculated with eq.(22) while the pluses show the results calculated with eq.(23). It is found that both are in excellent agreement with each other. That is, the use of the matrix elements of the momentum operator $\boldsymbol{p}$ instead of those of the Dirac matrices $\boldsymbol{\alpha}$ is very good approximation in calculating the diagonal optical conductivity.

We next show the off-diagonal optical conductivity, $\sigma_{x y}(\omega)$, of Fe in Fig. 4 and that of $\mathrm{Ni}$ in Fig. 5. Figures 4(a) and 5(a) represent $\omega \operatorname{Re}\left[\sigma_{x y}(\omega)\right]$ and Figs. 4(b) and 5(b) represent $\omega \operatorname{Im}\left[\sigma_{x y}(\omega)\right]$. Also, in these figures, the solid lines show the results calculated with eq.(22) while the pluses show the results calculated with eq.(23). It is found that there is a noticeable difference between the former and the latter although the difference is not very significant.

Finally, we show the Kerr spectra of Fe in Fig. 6 and those of Ni in Fig. 7. Figures 6(a) and $7(\mathrm{a})$ represent the Kerr rotation angle $\theta_{\mathrm{K}}(\omega)$ and Figs. 6(b) and 7(b) represent the Kerr ellipticity $\eta_{\mathrm{K}}(\omega)$. Also, in these figures, the solid lines show the results calculated with eq.(22) while the pluses show the results calculated with eq.(23). It is found that there is also a noticeable difference between the former and the latter although the difference is not very significant. In particular, the difference is considerable in the spectra of the Kerr rotation angle.

We thus arrive at the following conclusion. The approximation in which the matrix elements of the momentum operator $\boldsymbol{p}$ are used instead of those of the Dirac matrices $\boldsymbol{\alpha}$ is 

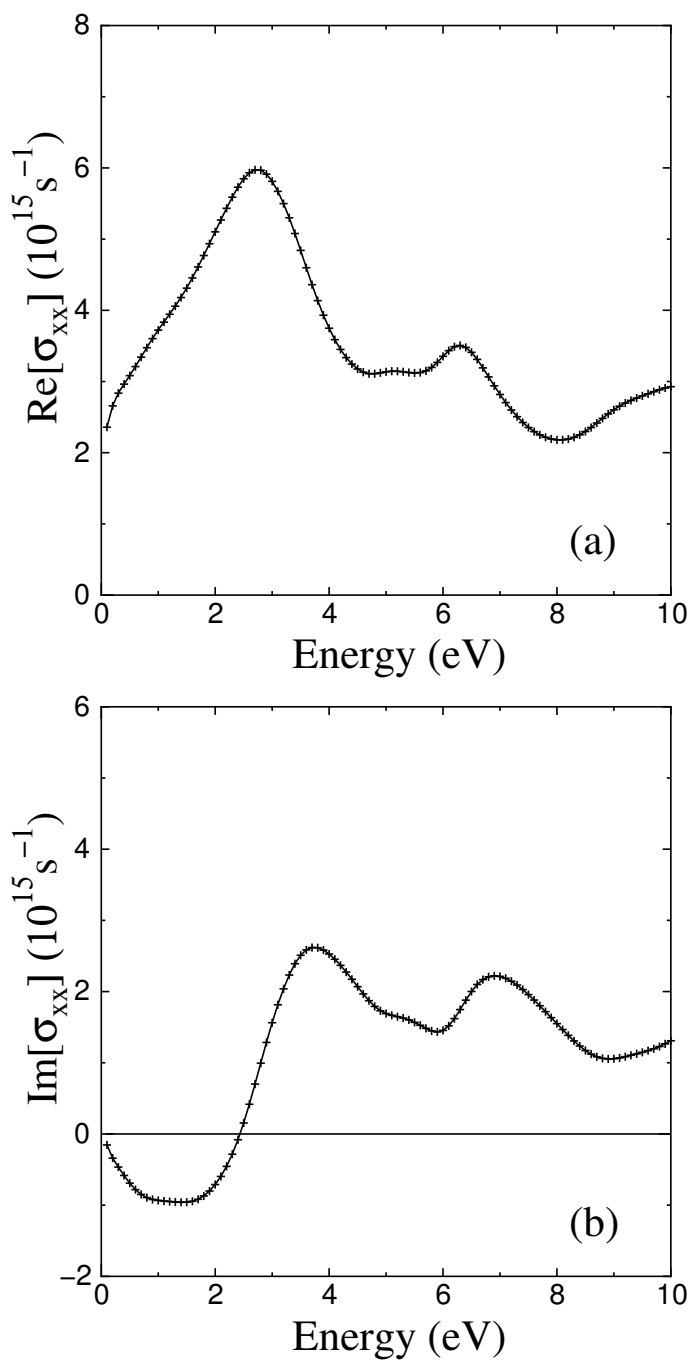

Fig. 2. Calculated diagonal optical conductivity for bcc Fe: (a) real part and (b) imaginary part. The solid lines are the results of fully relativistic calculations in which the matrix elements of the Dirac matrices $\boldsymbol{\alpha}$ are used and the pluses are those in which the matrix elements of the momentum operator $\boldsymbol{p}$ are used.

expected to be a good approximation in calculating physical quantities involving only the diagonal optical conductivity. On the other hand, it is likely that this approximation is not very good in calculating physical quantities involving the off-diagonal optical conductivity such as the Kerr rotation angle.

The reason why this approximation is not very good for the off-diagonal optical conductivity although it is good for the diagonal optical conductivity may be explained as follows. The off-diagonal optical conductivity vanishes unless spin-orbit coupling is taken into account. On the contrary, the diagonal optical conductivity is almost unchanged regardless of whether spinorbit coupling is taken into account or not. Moreover, the magnitude of the diagonal optical conductivity is about 100 times larger than that of the off-diagonal optical conductivity. It is 

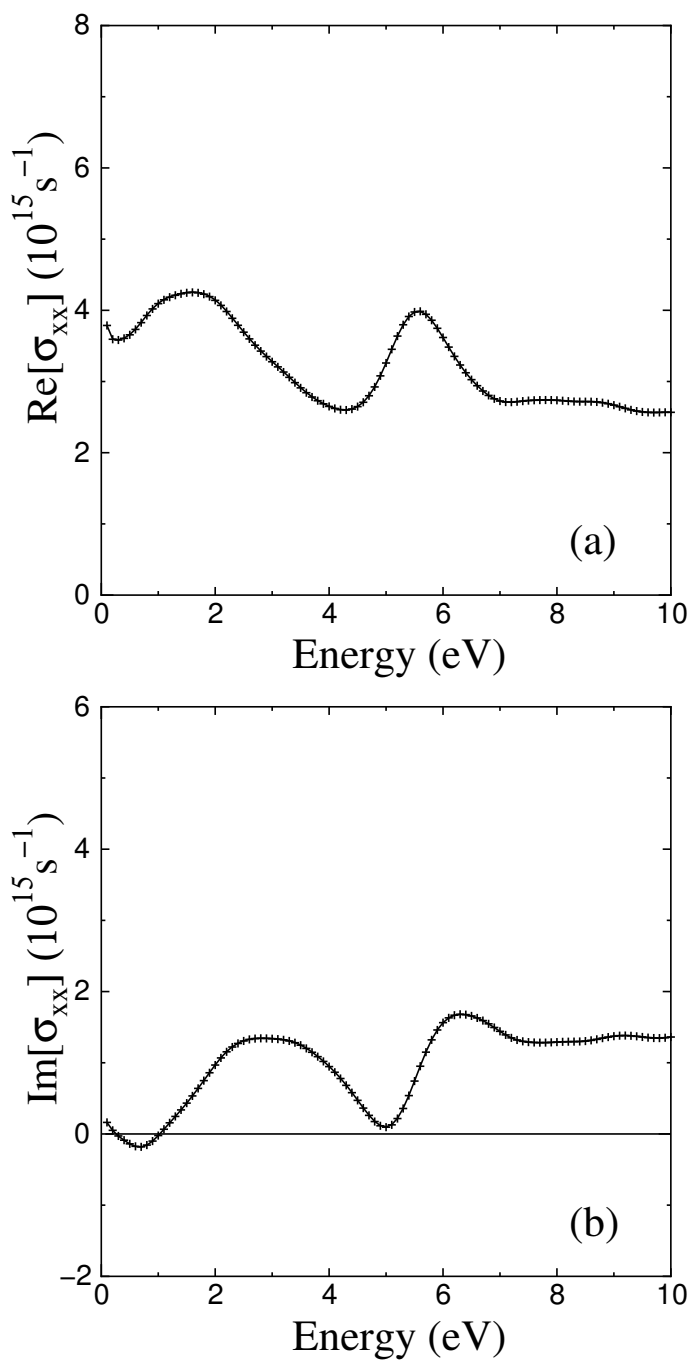

Fig. 3. Calculated diagonal optical conductivity for fcc Ni: (a) real part and (b) imaginary part. The solid lines are the results of fully relativistic calculations in which the matrix elements of the Dirac matrices $\boldsymbol{\alpha}$ are used and the pluses are those in which the matrix elements of the momentum operator $\boldsymbol{p}$ are used.

thus most likely that the off-diagonal optical conductivity is much more sensitive to spin-orbit coupling than the diagonal optical conductivity. On the other hand, the use of the matrix elements of the momentum operator $\boldsymbol{p}$ instead of those of the Dirac matrices $\boldsymbol{\alpha}$ inevitably neglects the terms of the same origin as spin-orbit coupling, e.g., $\left(\hbar / 4 m c^{2}\right)[\boldsymbol{\sigma} \times \nabla V(\boldsymbol{r})]$ with $\boldsymbol{\sigma}$ being the Pauli matrices. ${ }^{21)}$ Accordingly, this approximation can be worse for the off-diagonal optical conductivity than for the diagonal optical conductivity.

Finally, we compare the results of the present study to those of experimental and other theoretical studies. We first examine the Kerr spectra of Fe. The measured spectra of the Kerr rotation angle have several extremal points: -0.13 degree at $2.7 \mathrm{eV},-0.28$ degree at $4.5 \mathrm{eV}$, and 0.24 degree at $6.2 \mathrm{eV}{ }^{38)}$ Corresponding extremal points obtained in the present 

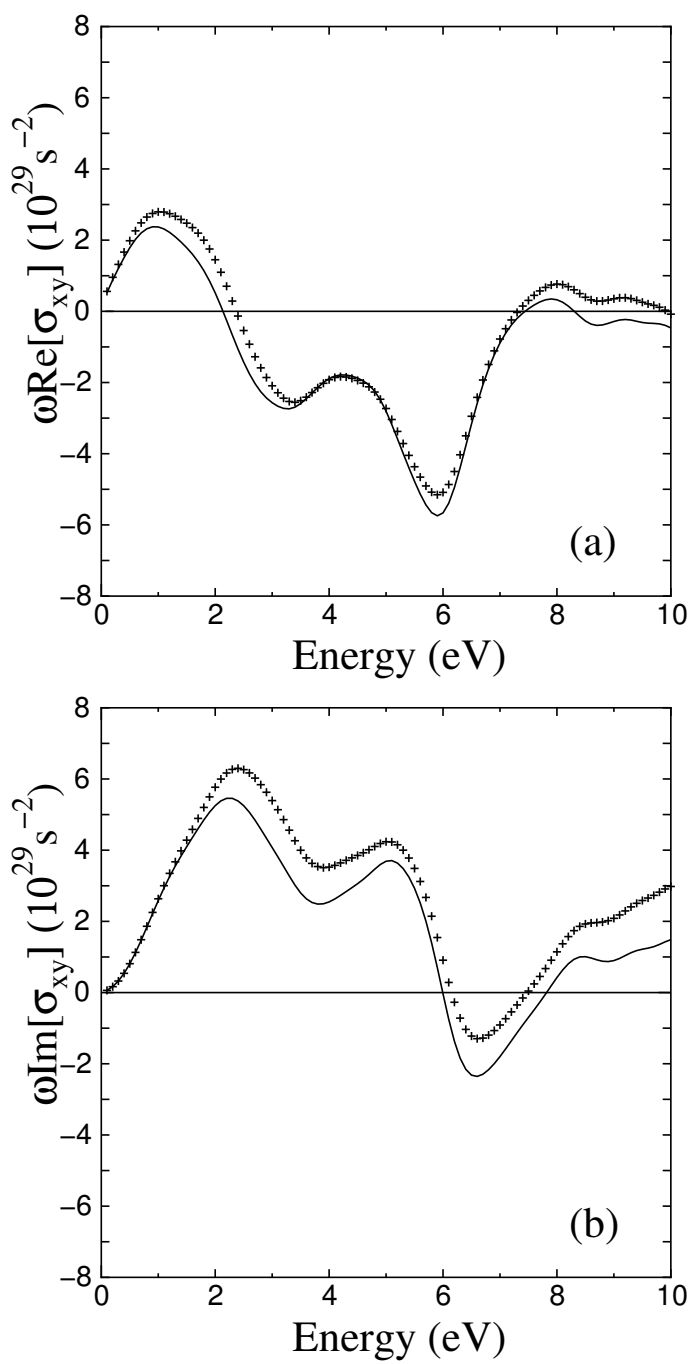

Fig. 4. Calculated off-diagonal optical conductivity for bcc Fe: (a) real part and (b) imaginary part. The solid lines are the results of fully relativistic calculations in which the matrix elements of the Dirac matrices $\boldsymbol{\alpha}$ are used and the pluses are those in which the matrix elements of the momentum operator $\boldsymbol{p}$ are used.

study are -0.14 degree at $3.2 \mathrm{eV},-0.30$ degree at $4.9 \mathrm{eV}$, and 0.18 degree at $6.3 \mathrm{eV}$. Also, the measured spectra of the Kerr ellipticity have several extremal points: -0.45 degree at $2.3 \mathrm{eV}$, -0.28 degree at $3.8 \mathrm{eV},-0.62$ degree at $5.5 \mathrm{eV}$, and -0.06 degree at $7.3 \mathrm{eV}$. Corresponding extremal points obtained in the present study are -0.34 degree at $1.7 \mathrm{eV},-0.12$ degree at $4.1 \mathrm{eV},-0.50$ degree at $5.7 \mathrm{eV}$, and 0.04 degree at $7.9 \mathrm{eV}$. We thus find that the calculated Kerr spectra in the present study are in good agreement with the measured spectra. Also, the agreement is better than the calculated spectra reported previously. ${ }^{8,24-26)}$

We next examine the Kerr spectra of Ni. The measured spectra of the Kerr rotation angle have several extremal points: -0.14 degree at $1.6 \mathrm{eV},-0.12$ degree at $2.3 \mathrm{eV}, 0.12$ degree at 3.3 $\mathrm{eV}$, and 0.18 degree at $5.1 \mathrm{eV}{ }^{39)}$ Corresponding extremal points obtained in the present study 

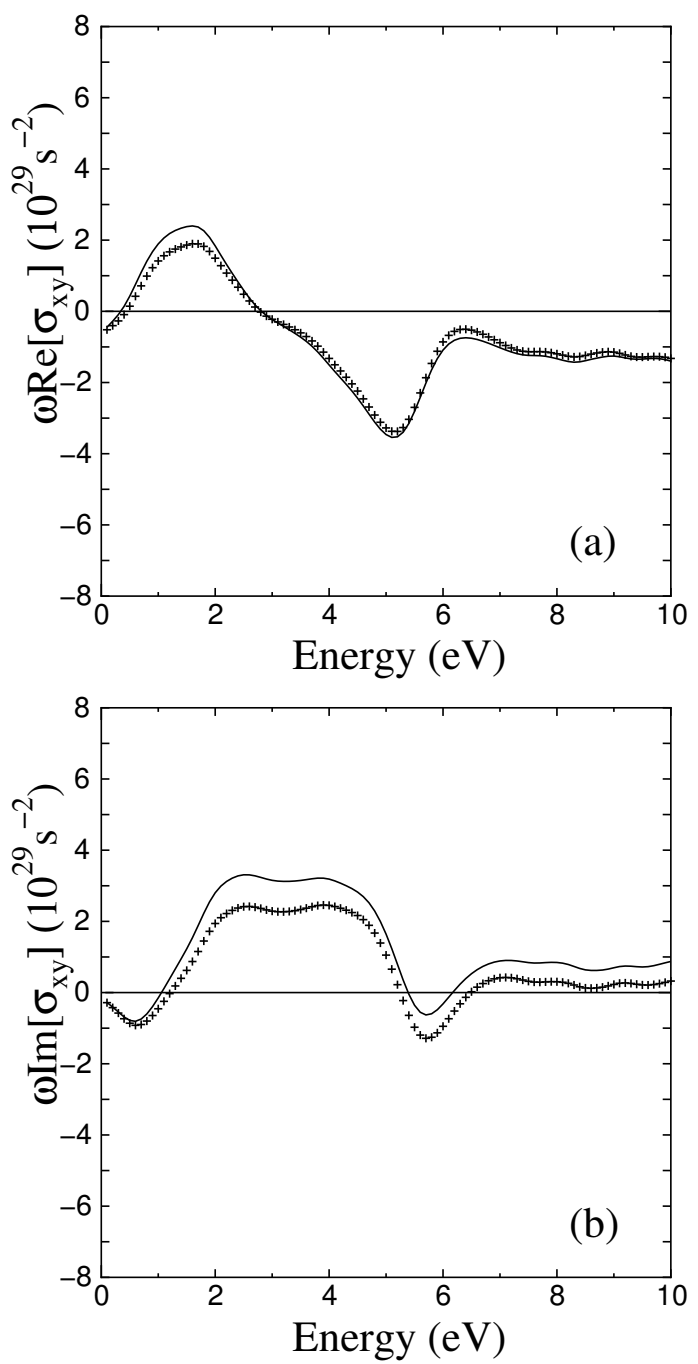

Fig. 5. Calculated off-diagonal optical conductivity for fcc Ni: (a) real part and (b) imaginary part. The solid lines are the results of fully relativistic calculations in which the matrix elements of the Dirac matrices $\boldsymbol{\alpha}$ are used and the pluses are those in which the matrix elements of the momentum operator $\boldsymbol{p}$ are used.

are -0.36 degree at $2.5 \mathrm{eV},-0.35$ degree at $3.1 \mathrm{eV},-0.38$ degree at $3.8 \mathrm{eV}$, and 0.16 degree at $5.2 \mathrm{eV}$. Also, the measured spectra of the Kerr ellipticity have several extremal points: 0.17 degree at $1.0 \mathrm{eV},-0.19$ degree at $4.2 \mathrm{eV}$, and 0.03 degree at $6.2 \mathrm{eV}$. Corresponding extremal points obtained in the present study are 0.27 degree at $0.70 \mathrm{eV},-0.51$ degree at $4.5 \mathrm{eV}$, and -0.06 degree at $6.2 \mathrm{eV}$. The Kerr spectra of Ni calculated in the present study are not in good agreement with the measured spectra; our results are larger than the experimental results and also larger than the results of other theoretical studies. ${ }^{8,25,26)}$

A possible reason for the good agreement in the case of $\mathrm{Fe}$ and the disagreement in the case of $\mathrm{Ni}$ is that many-body effects such as spin fluctuation are significant in Ni than in $\mathrm{Fe}$ because the magnetic moment per atom in $\mathrm{Ni}, 0.6 \mu_{\mathrm{B}}$, is considerably smaller than that in Fe, 

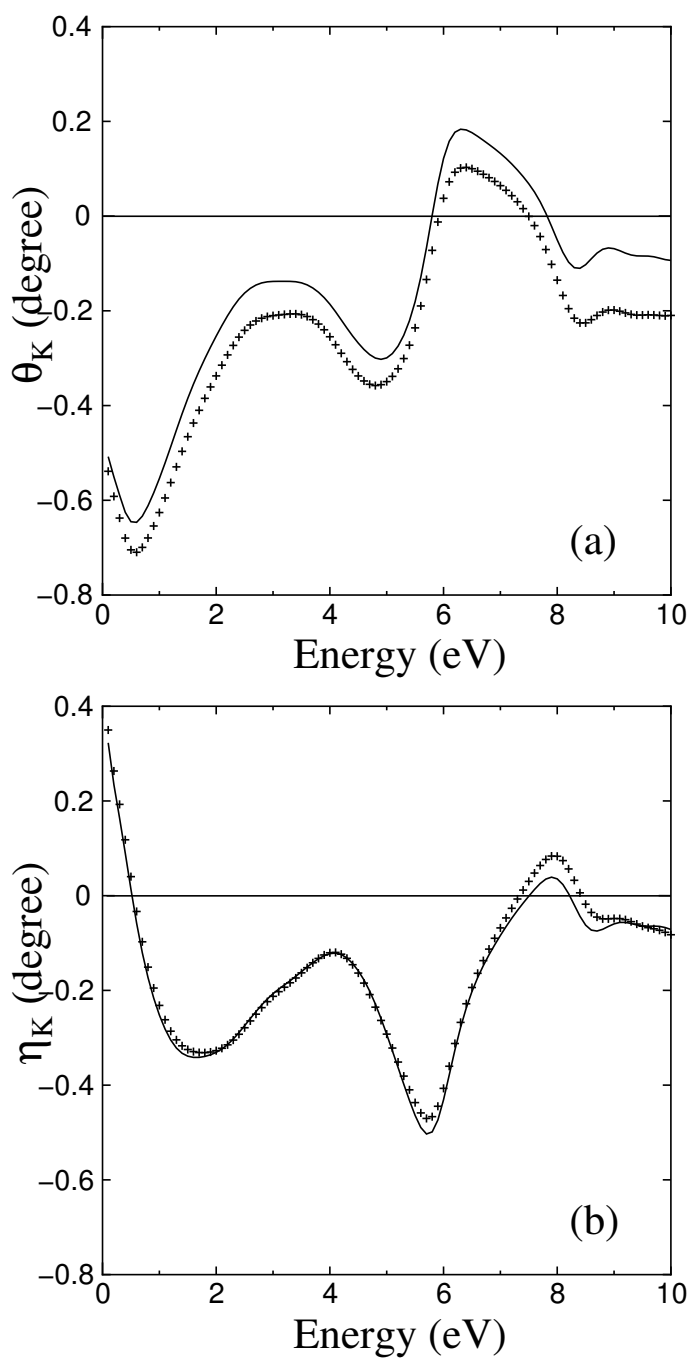

Fig. 6. Calculated Kerr spectra for bcc Fe: (a) Kerr rotation angle and (b) Kerr ellipticity. The solid lines are the results of fully relativistic calculations in which the matrix elements of the Dirac matrices $\boldsymbol{\alpha}$ are used and the pluses are those in which the matrix elements of the momentum operator $\boldsymbol{p}$ are used.

$2.2 \mu_{\mathrm{B}}$; it has been shown experimentally that there are variations of the Kerr spectra of Ni with temperature, ${ }^{40)}$ indicating the importance of spin fluctuation. We believe that, for $\mathrm{Ni}$, the agreement becomes better if measurements are carried out at lower temperatures.

\section{Conclusions}

We have studied the MOKE using fully relativistic calculations. Solving the Dirac equation directly, spin-orbit coupling is taken into account exactly and the matrix elements of the Dirac matrices $\boldsymbol{\alpha}$ are used in the fully relativistic expression of the Kubo formula for the optical conductivity derived with the relativistic sum rule. We have also carried out calculations using the partly relativistic expression in which the matrix elements of the momentum operator $\boldsymbol{p}$ 

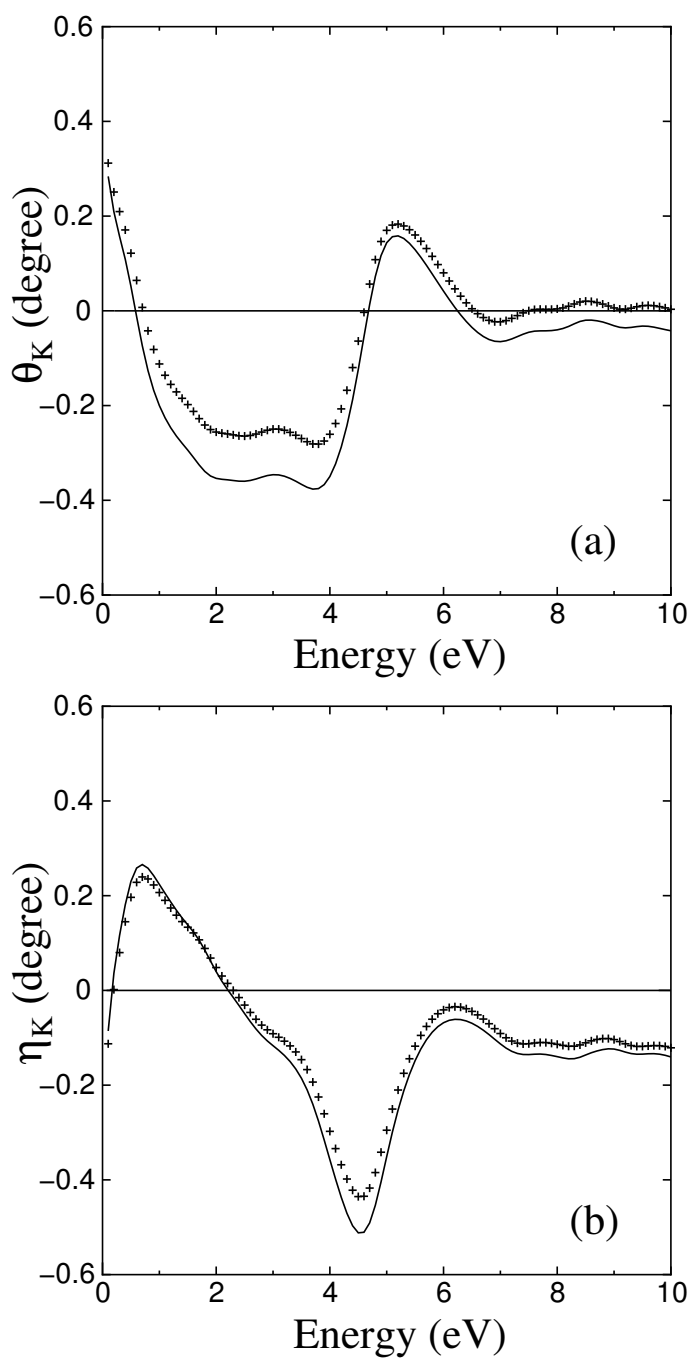

Fig. 7. Calculated Kerr spectra for fcc Ni: (a) Kerr rotation angle and (b) Kerr ellipticity. The solid lines are the results of fully relativistic calculations in which the matrix elements of the Dirac matrices $\boldsymbol{\alpha}$ are used and the pluses are those in which the matrix elements of the momentum operator $\boldsymbol{p}$ are used.

are used instead. As an example, we have performed calculations for bcc Fe and fcc $\mathrm{Ni}$ and have found that the partly relativistic treatment is good for the diagonal optical conductivity while it is not very good for the off-diagonal optical conductivity, the Kerr rotation angle, and the Kerr ellipticity. Finally, we have compared the results of the present study to those of experimental and other theoretical studies and have found that the agreement with the measured spectra is good for Fe while it is not good for Ni. A possible reason for this is that many-body effects, e.g., spin fluctuation, are more important in Ni than in Fe. 


\section{References}

1) M. H. Kryder: J. Appl. Phys. 57 (1985) 3913.

2) W. H. Meiklejohn: Proc. IEEE 74 (1986) 1570.

3) J. Schoenes: in Materials Science and Technology, ed. K. H. J. Buschow (VHC, New York, 1992) Vol. 3A, p. 147.

4) H. Ebert: Rep. Prog. Phys. 59 (1996) 1665.

5) P. M. Oppeneer, V. N. Antonov, A. N. Yaresko, A. Ya. Perlov, T. Kraft, and H. Eschrig: J. Magn. Soc. Jpn. 20 (1996) 41.

6) P. M. Oppeneer and V. N. Antonov: in Proceedings of Spin-orbit-influenced Spectroscopies of Magnetic Solids (Springer, Berlin, 1996) p. 29.

7) K. H. J. Buschow: in Ferromagnetic Materials, ed. E. P. Wohlfarth and K. H. J. Buschow (NorthHolland, Amsterdam, 1988) Vol. 4, Chap. 5.

8) P. M. Oppeneer, T. Maurer, J. Sticht, and J. Kübler: Phys. Rev. B 45 (1992) 10924.

9) P. M. Oppeneer, T. Kraft, and H. Eschrig: J. Magn. Magn. Mater. 148 (1995) 298.

10) P. M. Oppeneer, T. Kraft, and H. Eschrig: Phys. Rev. B 52 (1995) 3577.

11) H. Ebert and H. Akai: J. Appl. Phys. 67 (1990) 4798.

12) I. Osterloh, P. M. Oppeneer, J. Sticht, and J. Kübler: J. Phys.: Condens. Matter 6 (1994) 285.

13) J. Kübler: J. Phys. Chem. Solids 56 (1995) 1529.

14) P. M. Oppeneer, V. N. Antonov, T. Kraft, H. Eschrig, A. N. Yaresko, and A. Ya. Perlov: Solid State Commun. 94 (1995) 255.

15) P. M. Oppeneer, V. N. Antonov, T. Kraft, H. Eschrig, A. N. Yaresko, and A. Ya. Perlov: J. Appl. Phys. 80 (1996) 1099.

16) P. M. Oppeneer, V. N. Antonov, T. Kraft, H. Eschrig, A. N. Yaresko, and A. Ya. Perlov: J. Phys.: Condens. Matter 8 (1996) 5769.

17) T. Kusakabe, K. Kyuno, and S. Asano: J. Magn. Magn. Mater. 126 (1993) 535.

18) G. Y. Guo and H. Ebert: Phys. Rev. B 51 (1995) 12633.

19) J.-G. Ha, K. Kyuno, and R. Yamamoto: J. Phys.: Condens. Matter 8 (1996) 677.

20) S. Uba, L. Uba, A. N. Yaresko, Ya. A. Perlov, V. N. Antonov, and R. Gontarz: Phys. Rev. B 53 (1996) 6526.

21) T. Kraft, P. M. Oppeneer, V. N. Antonov, and H. Eschrig: Phys. Rev. B 52 (1995) 3561.

$22)$ P. M. Oppeneer, M. S. S. Brooks, V. N. Antonov, T. Kraft, and H. Eschrig: Phys. Rev. B 53 (1996) R10437.

23) A. N. Yaresko, P. M. Oppeneer, Ya. A. Perlov, V. N. Antonov, T. Kraft, and H. Eschrig: Phys. Rev. B 77 (1996) 5253.

24) H. Miyazawa and T. Oguchi: J. Magn. Magn. Mater. 192 (1999) 325.

25) A. Delin, O. Eriksson, and B. Johansson: Phys. Rev. B 60 (1999) 14105.

26) J. Kuneš and P. Novák: J. Phys.: Condens. Matter 11 (1999) 6301.

27) C. S. Wang and J. Callaway: Phys. Rev. B 9 (1974) 4897.

28) M. Singh, C. S. Wang, and J. Callaway: Phys. Rev. B 11 (1975) 287.

29) S. Suzuki and K. Nakao: J. Phys. Soc. Jpn. 68 (1999) 1982.

30) V. N. Antonov, A. I. Bagljuk, A. Ya. Perlov, V. V. Nemoshkalenko, Vl. N. Antonov, O. K. Andersen, and O. Jepsen: Low Temp. Phys. 19 (1993) 494. 
31) L. I. Schiff: Quantum Mechanics (McGraw-Hill, Tokyo, 1968).

32) D. M. Ceperley and B. J. Alder: Phys. Rev. Lett. 45 (1980) 566.

33) J. Perdew and Y. Wang: Phys. Rev. B 23 (1981) 5048.

34) A. D. Becke: J. Chem. Phys. 88 (1988) 2547.

35) B. Delley: J. Chem. Phys. 92 (1990) 508.

36) S. Suzuki and K. Nakao: J. Phys. Soc. Jpn. 66 (1997) 3881.

37) L.-K. Hua and Y. Wang: Applications of Number Theory to Numerical Analysis (Springer-Verlag, Berlin, 1981).

38) T. Katayama, N. Nakajima, N. Okusawa, Y. Miyauchi, T. Koide, T. Shidara, Y. Suzuki, and S. Yuasa: J. Magn. Magn. Mater. 177-181 (1998) 1251.

39) K. Nakajima, H. Sawada, T. Katayama, and T. Miyazaki: Phys. Rev. B 54 (1996) 15950.

40) G. Q. Di and S. Uchiyama: J. Appl. Phys. 75 (1994) 4270. 